\title{
解椭圆型问题的无限元方法
}

应隆安

(北京大学数学系, 北京 100871)

摘要

本文给了一个求解变系数湅圆型方程的无限元方法。在解具有奇性时可获得很 准确的奇性数值解, 而且计算量小, 不霹要事先知道方程解的奇性㜾及特解的解析表 达式. 最后给了一个数值例子, 将它与有隄元方法做了比较。

\section{关键词：无限 元方法,椭圆型方程,奇性,内边界}

自从无限元方法问世以来, 它已应用到很多椭圆型问题上. 首先有 Laplace 方程 ${ }^{[1]}$ 及线 性弹性问题 ${ }^{[2]}$, 后来又有 Stokes 方程 ${ }^{[3], 1)}$ 及 Helmholiz 方程 ${ }^{[4,5]}$ 等。当这些方程的解在某些点 具有奇性, 或者在无界区域上求解时, 用无限元方法都可以得到很准确的解. 与求解同类问题 的其他方法比较, 它有一个优点: 在求解时, 不需要知道方程的特解的解析表达式. 因此, 当 特解十分复杂, 很难写出解析表达式时,它就显得特别有效。

以前的无限元方法有一个局限性, 它强烈地依赖于方程的一个性质：在取定一个比例因 子后,作自变量的相似变换,则方程不变。这样,对于变系数问题, 或者方程有一些低阶项时, 应用无限元方法就有困难, 至少不能把无限阶代数方程精确地归结为一个有限阶问题. 文献 [4]中处理的 Helmholtz 方程是一个例外, 但那里的方法依赖于解对于一个参数的解析性, 不 易推广,计算时也不如原方法那样简浯.

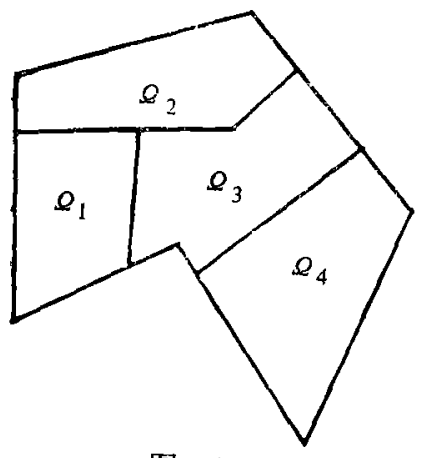

图 1

本文的目的是给出一个无限元方法，它可用于一般的 椭圆型方程。虽然我们在叙述时以一个二阶线性方程的 Dirichlet 边值问题为例，但是这个方法也可以用于高阶方 程. 在求解非线性问题时, 每一步逼近只要是解一个线性 句题,也可以用本方法. 本方法还有希望推广到求解发展 型方程上去.

\section{一、解 的 奇 性}

我们以一个凹角及内边界问题为例, 以 $x$ 记平面 $\mathrm{R}^{2}$ 上 的点, 设 $Q$ 是 $\mathrm{R}^{2}$ 上一一个多边形区域。在 $Q$ 中又有有限条折 线把它分割为有限个多边形子区域 $Q_{l}, l=1, \cdots, L$ (图 1). 设 $a_{i j}(x), 1 \leqslant i, j \leqslant 2$, 是

本文 1990 年 8 月 11 日收到。

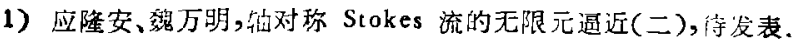


$C^{1}(\bar{\Omega})$ 中函数, $a_{21}(x)=a_{12}(x)$, 满足椭圆型条件

$$
\sum_{i, j=1}^{2} a_{i j}(x) \xi_{i} \xi_{j} \geqslant \alpha|\xi|^{2}, \quad \forall \xi \in \mathbb{R}^{2},
$$

其中常数 $\alpha>0$, 设函数 $p(x)$ 在每一子区域 $\Omega_{l}$ 上取正的常数值, 函数 $a_{0}(x)$ 在 $Q$ 上有界、非 负, $f \in L^{2}(\Omega)$. 考虑如下变分问题: 求 $u \in H_{0}^{1}(\Omega)$,使

$$
\int_{\Omega}\left(\sum_{i, j=1}^{2} a_{i j} p \frac{\partial u}{\partial x_{i}} \frac{\partial v}{\partial x_{j}}+a_{0} u v\right) d x=\int_{0} f v d x, \forall v \in H_{0}^{1}(\Omega),
$$

本文采用通常的 Sobolev 空间的记号, 即以 $H^{s}(\Omega), H_{0}^{s}(\Omega)$ 记空间, 以 \|\|$_{s}$ 记范数. 由 LaxMilgram 定理容易证明问题(1.1)有唯一解.

$\boldsymbol{u}$ 可能在以下各种点上有奇性: 内边界线交点、内边界线转折点、内边界线与区域边界 $\partial Q$ 的交点、边界 $\partial \Omega$ 上内角大于 $\pi$ 的点. 在这些点附近, 解的一阶微商可能无界. 以后我们 将这些点统称为奇点.

关于上述问题解的奇性, 如今理论已十分完整. 但是为了下面求解的需要, 我们用稍微不 同的形式进行叙述.

人所共知,解 $u$ 在每一子区域 $\Omega_{l}$ 上满足方程

$$
-\sum_{i, j=1}^{2} \frac{\partial}{\partial x_{i}}\left(a_{i i}(x) p(x) \frac{\partial u}{\partial x_{i}}\right)+a_{0}(x) u=f(x),
$$

在边界 $\partial Q$ 上满足条件

在内边界上满足条件

$$
\left.u\right|_{\partial Q}=0
$$

$$
\left\{\begin{array}{l}
u \text { 在内边界上连续, } \\
p \sum_{i, j=1}^{2} a_{i} n_{i} \frac{\partial u}{\partial x} \text { 在内边界上连续, }
\end{array}\right.
$$

其中 $n_{i}$ 表示内边界上单位法向量的分量.

以 $x^{(m)}, m=1, \cdots, M$, 表示奇点, 我们以 $\Omega^{(m)}$ 表示包含 $x^{(m)}$ 且不包含其他奇点的一个邻 域, 记 $Q^{*}=Q \backslash \bigcup_{m=1}^{n} \bar{\Omega}^{(m)}$, 则有

定理 1. $u$ 在每一个 $Q^{*} \cap Q_{z}$ 上属于 $H^{2}$, 在 $Q^{(m)}$ 上, $u=v+w . v \in H^{1}\left(\Omega^{(m)}\right)$, 满足方程

$$
-\sum_{i, i=1}^{2} \frac{\partial}{\partial x_{i}}\left(a_{i j}\left(x^{(m)}\right) p(x) \frac{\partial v}{\partial x_{i}}\right)=0,
$$

以及与(1.3)式相应的边界条件. $w$ 在每一个 $Q^{(m)} \cap Q_{l}$ 上属于 $H^{2}$.

证. 我们用文献中的结果. 在文献[6]中已证 $u$ 在 $\Omega^{*} \cap \Omega_{l}$ 上属于 $H^{2}$. 下面在一个子 区域 $Q^{(m)}$ 中讨论.

由文献 [6], 只要适当取 $Q^{(m)}$, 就有一个一一的 $C^{1}$ 映射 $F: x \rightarrow y$, 将 $Q^{(m)}$ 映为 $y$ 平面上零 点的一个邻域, $y\left(x^{(m)}\right)=0 . F$ 在除了 $x^{(m)}$ 点以外属于 $C^{2}$, 而且二阶导数一致有界. 每条 由 $x^{(m)}$ 出发的内边界线与边界线都映为 $y$ 平面上的直线. 在此变换下, $u(x(y))=v_{1}(y)+$ $w_{1}(y) . v_{1} \in H^{1}$,是方程

$$
-\nabla\left(p(x(y)) \nabla v_{1}\right)=0
$$


的㐷解. $w_{1}(y)$ 在每个子区域上属于 $H^{2}$.

令 $G: x \rightarrow y$ 为 $F$ 在 $x^{(m)}$ 点的 Frechet 微商, 则因为 $G$ 是线性映射, 它也映上述内边界线与 江界线为直线, 并且在 $y=0$ 处两族直线的斜率一致, 于是这些直线重合, 即映射 $F$ 与 $G$ 将 $Q^{(m)} \cap \Omega_{l}$ 映为相同的于区域。

由文献 [6],变换 $F$ 将方程(1.2) 在边界线及内边界线上变为方程 (1.6), 由于变换 $G$ 为上述 变换的线性主要部分, 不难看出, 它将方程 (1.5)在整个区域上变为方积 $(1.6)$. 于是函数 $v_{\mathrm{i}} \circ G$ 在 $Q^{(m)}$ 上满足方程 (1.5). 因为 $w_{1} \circ F$ 在每个子区域上都蝺于 $H^{2}$, 为证明本定理, 我们只要证 明, 在每个子区域上 $v_{1} \circ F-v_{1} \circ G$ 也属于 $H^{2}$ 就够了.

由文献 [6], 在极坐标下, $v_{1}$ 是形如 $r^{a} \Theta(\theta)$ 的函数的有限和, 其中 $a<1, \Theta(\theta)$ 是相应的 Sturm-Liouville 问题的解, 它是分段充分光滑的. 又因为 $\nu_{1} \in H^{1}$, 所以 $a>0$. 我们计算二 阶微商

$$
\begin{aligned}
& \frac{\partial^{2}}{\partial x_{i} \partial x_{i}} v_{1}(F(x))=\sum_{k, l} \frac{\partial^{2} v_{1}}{\partial y_{k} \partial y_{l}} \frac{\partial y_{k}}{\partial x_{i}} \frac{\partial y_{l}}{\partial x_{j}}+\sum_{k} \frac{\partial v_{1}}{\partial y_{k}} \frac{\partial^{2} y_{k}}{\partial x_{i} \partial x_{j}}, \\
& \frac{\partial^{2}}{\partial x_{i} \partial x_{j}} v_{1}(G(x))=\sum_{k, l} \frac{\partial^{2} v_{1}}{\partial z_{k} \partial z_{l}} \frac{\partial z_{k}}{\partial x_{i}} \frac{\partial z_{l}}{\partial x_{j}},
\end{aligned}
$$

其中 $y=F(x), z=G(x)$. 由 $F$ 的光滑性

$$
|y-z| \leqslant C r^{2},\left|\frac{\partial y_{k}}{\partial x_{i}}-\frac{\partial z_{k}}{\partial x_{i}}\right| \leqslant C r,
$$

再利用 $v_{1}$ 的表达式得

$$
\begin{aligned}
& \left|\frac{\partial^{2} y_{1}}{\partial y_{k} \partial y_{l}}\right| \leqslant C r^{a-2},\left|\frac{\partial^{2} v_{1}}{\partial z_{k} \partial z_{l}}\right| \leqslant C r^{a-2}, \\
& \left|\frac{\partial^{2} v_{1}}{\partial y_{k} \partial y_{l}}-\frac{\partial^{2} v_{1}}{\partial z_{k} \partial z_{l}}\right| \leqslant C r^{a-3}|y-z| .
\end{aligned}
$$

于是

$$
\left|\frac{\partial !}{\partial x_{1} \partial x_{i}} v_{1}(F(x))-\frac{\partial^{2}}{\partial x_{i} \partial x_{j}} v_{1}(G(x))\right| \leqslant C r^{a-1}
$$

它属于 $L^{2}$. 定理证毕.

\section{二、无限元方法}

本节叙述求问题 (1.1) 数值解的无限元方法. 以线性三角形单元为例. 取每一个奇点的 邻域 $Q^{(m)}$ 为多边形, 并且 $Q^{(m)}$ 关于 $x^{(m)}$ 点是星形的, 即联结 $Q^{(m)}$ 内每一点与 $x^{(m)}$ 的直线段都 整个地在 $Q^{(m)}$ 内. 以 $\partial Q^{(m)}$ 记 $\Omega^{(m)}$ 的边界. 在 $\Omega^{(m)}$ 中, 按照定理 1 的启发, 作两种剖分. 其 一为无限元剖分, 它给出方程(1.5)的解 $v$ 的一个近似, 其二为通常的有限元剖分, 它给出 $w$ 的 一个近似(图 2). 在 $\Omega^{*}=\Omega \backslash \bigcup_{m=1} \dot{Q}^{(m)}$ 上作通常的有限元剖分. 我们要求这些剖分互相协 调, 即在 $\partial Q^{(m)}$ 上它们的节点是一致的. 在 $Q^{(m)}$ 上以两套网格的函数的和作为近似解.

关于无限元剖分, 很多论文中已有描述. 为完整起见, 重述如下: 取一个常数 $\xi \in(0,1)$, 以 $\boldsymbol{x}^{(m)}$ 为相似中心, 以 $5^{2}, \cdots, 5^{k}, \cdots$ 为比例常数, 作 $\partial Q^{(m)}$ 的相似形. 于是 $\Omega^{(m)}$ 被分割为 无限多“层”, 在每一层内作三角北剖分. 要求各层的剖分皆几何相似, 而且整个区域 $\Omega^{(m)}$ 的 

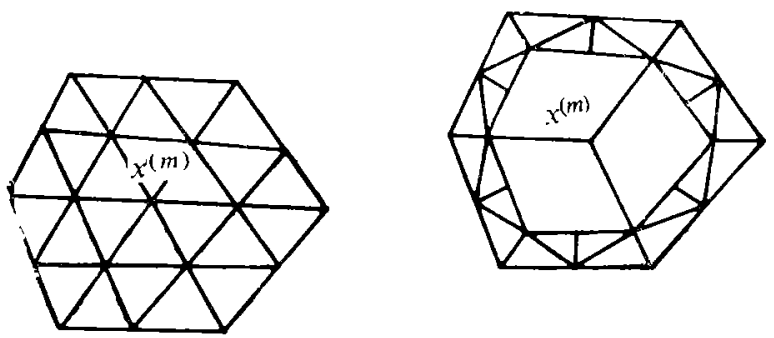

图 2

剖分是正规的.

以 $y_{0}, \cdots, y_{k}, \cdots$ 记各多边形上节点值作成的向量, 则利用方程, 当 $y_{0}$ 给定以后, $y_{1}, \cdots$, $y_{k}, \cdots$ 都被它唯一确定. 在计算中, 起关键作用的是两个矩阵.一个是组合刚度矩阵 $K_{z}$, 它是 对称半正定的, 当 $y_{0}$ 给定后, 在 $Q^{(m)}$ 上的“应变能”就是 $\frac{1}{2} y_{0}^{\top} K_{z} y_{0}$, 其中 $T$ 表示转置. 另一个 是转移矩阵 $X$, 它的作用表现在如下关系式中:

$$
y_{k}=X^{k} y_{0}, k=1,2, \cdots \text {. }
$$

已有很多工作研究了 $K_{x}$ 与 $X$ 的求法, 读者可以参看文献 $[2,7,8]$.

对于 $Q^{(m)}$ ，我们作两个 $H^{(}\left(Q^{(m)}\right)$ 的有限维子空间 $V^{(m)}$ 与 $W^{(m)}, V^{(m)}$ 对应于无限元剖 分, 其中节点值 $y_{0}$ 为任意, $y_{k}, k=1,2, \cdots$, 由(2.1)式确定, $W^{(m)}$ 是对应于有限元剖分的常规 有限元子空间. 在区域 $\Omega^{*}$ 上也作有限元子空间 $U^{*}$. 最后, 我们作 $H_{0}^{1}(Q)$ 的有限维子空间 $U_{h}$, 当 $u \in U_{k}$, 则 $u$ 在 $\Omega^{*}$ 上的躡制属于 $U^{*}$, 在 $Q^{(m)}$ 上, $u=v+w, v \in V^{(m)}, w \in W^{(m)}$.

无限元解 $u_{h} \in U_{h}$ 满足

$$
\int_{\Omega}\left(\sum_{i, j=1}^{2} a_{i j} p \frac{\partial u_{h}}{\partial x_{i}} \frac{\partial v}{\partial x}+a_{0} u_{\xi} \nu\right) d x=\int_{\varrho} f v d x, \forall v \in U_{h} .
$$

容易证明, 它也有唯一解.

下面证明无限元方法的收玫性。为此, 引进加权的 Sobolev 空间. 令

$$
\zeta(x)=\prod_{m=1}^{M}\left|x-x^{(m)}\right| \text {. }
$$

总间 $H^{2(\sigma)}(Q)(\sigma<2)$ 的定义为

$$
\left\{u ;\|u\|_{2(\sigma), \Omega}=\left[\|u\|_{1, \Omega}^{2}+\sum_{l=1}^{L} \int_{\Omega_{l}} \zeta^{2-\sigma}\left|\partial^{2} u\right|^{2} d x\right]^{1 / 2}<+\infty\right\},
$$

其中 $\left|\partial^{2} u\right|=\sum_{a+\beta=2}\left|\frac{\partial^{2} u}{\partial x_{\imath}{ }^{\prime} \partial x_{2}^{\beta}}\right|$.

设有一族剖分, 它们的单元最大直径 $h$ 趋于零, 并且区域 $\boldsymbol{Q}^{(m)}$ 与剖分无关. 我们假设剖 分是正规的,即所有单元内角有公共下界 $\theta_{0}>0$. 以 $C$ 记通用常数, 则有

定理 2. 在上述假设条件下, 无限元解的误差有如下估计:

$$
\left\|u-u_{h}\right\|_{\imath, \Omega} \leqslant C h\|f\|_{0, \Omega} \text {. }
$$

证. 首先我们证明, 对于问题 (1.1) 的解 $u$, 存在 $v \in(0,2)$, 使

$$
\|\boldsymbol{u}\|_{2(, 5,3,} \leqslant C\|f\|_{0, \Omega},
$$


并且在每个区域 $Q^{(m)}$.上, 由定理 1 确定的函数 $w$ 满足

$$
\|w\|_{2, \Omega^{(m)} \cap \Omega_{l}} \leqslant C\|f\|_{0, \Omega} \text {. }
$$

先证明(2.4)式. 由文献 $[6], u=v_{1}+w_{1}$, 其中 $w_{1}$ 满足

$$
\left\|w_{1}\right\|_{2, \Omega_{l}} \leqslant C\|f\|_{0 . \Omega} \text {, }
$$

因此我们只要估计 $v_{1}$ 。按文献 $[6], v_{1}$ 属于一有限维空间, 并且

$$
\left\|L v_{1}\right\|_{0, \Omega} \leqslant C\|f\|_{0, \Omega} \text {, }
$$

其中 $L$ 是由问题（1.1）确定的二阶椭圆型算子. 在每一个奇点处, $\nu_{1}$ 的二阶导数有奇性阶 $\left|x-x^{(m)}\right|^{a-2}$, 因此只要取 $0<\sigma<? a$, 就有 $v_{1} \in H^{2(n)}(D)$. 又, 有限维空间上范数皆等价, 所 以从(2.7)式得

$$
\left\|v_{1}\right\|_{2(\sigma), s} \leqslant C\left\|_{1}\right\|_{0.2}
$$

于是(2.4)式已经得到了证明. 我们再证明(2.5)式。由定理 1, $w$ 由两部分组成, 其中一部分 为 $w_{1}$, 它的估计已由(2.6) 式给出, 另一部分为 $v_{1} \circ F-v_{1} \circ G$, 由(2.8)式得

$$
\left\|\nu_{1} \circ F-\nu_{1} \circ G\right\|_{0,0}(m) \leqslant C\left\|\nu_{1}\right\|_{2(s), 0} \leqslant C\|f\|_{0, \varrho} \text {. }
$$

但 $v_{1}$ 属于有限维空间, 由有限维空间上范数的等价性即得

$$
\left\|v_{1} \circ F-v_{1} \circ G\right\|_{2 . e^{(m)} n \Omega_{l}} \leqslant C\|f\|_{0 . \Omega} \text {. }
$$

(2.5)式也已经得到了证明.

我们在 $W^{(n)}$ 中作 $w$ 的插值函数 $w_{l}$, 则有 ${ }^{[9]}$

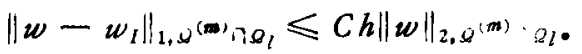

再在 $\partial Q^{(m)}$ 上作 $v$ 的插值函数,得到一个节点值向量 $y_{0}$, 然后利用(2.1)式确定 $y_{k}$, 于是可得一 函数 $v_{I} \in V^{(m)}$. 在文献 [10]中已经证明

$$
\left\|v-v_{I}\right\|_{1, Q^{(m)}} \leqslant C h\|v\|_{2(\sigma), S^{(m)}} .
$$

令 $u_{I}=v_{i}+w_{I}$. 在 $Q^{*}$ 上也作插值函数 $u_{I}$, 也有

$$
\left\|u-u_{l}\right\|_{1, s^{*}} \leqslant C h\|u\|_{2,8 *} \text {. }
$$

不难看出, $u_{l} \in U_{h}$,由(2.4),(2.5),(2.9)-(2.11)式得

$$
\left\|u-u_{l}\right\|_{1 . s} \leqslant C h\|f\|_{0, o} .
$$

无限元方法的抽象误差估计与有限元方法一样 ${ }^{[9]}$

$$
\left\|u-u_{h}\right\|_{l, \rho} \leqslant C \operatorname{linf}_{\nu \in U_{h}}\|u-v\|_{1,0}
$$

合并(2.12),(2.13)式即得(2.3)式. 证毕.

对于 $L^{2}$ 模, 还有如下估计:

定理 3. 在定理 2 的条件下, 有

$$
\left\|u-u_{k}\right\|_{0 . \Omega} \leqslant C h^{2}\|f\|_{0.8} \text {. }
$$

它的证明方法是经典的对偶分析 ${ }^{[9]}$, 并且利用估计式 (2.4), (2.5), 此处从略.

\section{三、刚度矩阵的计算}

在求解 (2.2)式时,一般需要计算一些数值积分. 有些被积函数不是光滑的, 甚至不是连 续的, 在奇点附近还是无界的. 因此,能否准确地计算这些积分, 是无限元方法的一个重要问 题. 
为叙述方便,我们仅讨论(2.2)式的主要部分

$$
\int_{\Omega^{(m)}} \sum_{i, j=1}^{2} a_{i} p \frac{\partial u}{\partial x_{i}} \frac{\partial v}{\partial x_{j}} d x, u, v \in V^{(m)}+W^{(m)} \text {. }
$$

它又可以分解为四种情况:
(a) $u \in V^{(m)}, v \in V^{(m)}$;
(b) $u \in V^{(m)}, v \in W^{(m)}$;
(c) $u \in W^{(m)}, v \in V^{(m)}$;
(d) $u \in W^{(m)}, v \in W^{(m)}$.

我们逐一说明它们的计算方法. 对于 (a), 可以写成

$$
\int_{\Theta^{(m)}} \sum_{i, j=1}^{2} a_{i j}\left(x^{(m)}\right) p \frac{\partial u}{\partial x_{i}} \frac{\partial v}{\partial x_{j}} d x+\int_{Q^{(m)}} \sum_{i, j=1}^{2}\left(a_{i j}(x)-a_{i j}\left(x^{(m)}\right)\right) p \frac{\partial u}{\partial x_{i}} \frac{\partial v}{\partial x_{i}} d x,
$$

其中第一项就是组合刚度矩阵 $K_{z}$, 它的计算方法是已知的. 第二项中有一个因子 $a_{i j}(x)-$ $a_{i j}\left(x^{(m)}\right)$, 它使被积函数在 $Q^{(m)}$ 上有界. 可以用通常的数值积分公式. 对于(b), 由于 $v \in W^{(m)}$, 可以按有限元剖分的单元逐一计算, 以 $K$ 记一个单元, 则由 Green 公式得

$$
\begin{aligned}
\int_{K} \sum_{i, j=1}^{2} a_{i j} p \frac{\partial u}{\partial x_{i}} \frac{\partial v}{\partial x_{j}} d x \\
=-\int_{K} \sum_{i, j=1}^{2} \frac{\partial a_{i j}}{\partial x_{i}} p u \frac{\partial v}{\partial x_{i}} d x+\int \sum_{\partial K}^{2} a_{i, j} p u n_{i} \frac{\partial v}{\partial x_{i}} d s,
\end{aligned}
$$

其中我们利用了 $p, \frac{\partial v}{\partial x_{j}}$ 均为常值. 右端两个积分的被积函数都是连续有界的, 可以用通常 的数值积分公式. (c)与(b)是一样的, 由对称性, 可以不计算. (d) 就是通常的有限元方法的 刚度矩阵计算. 计算皇元刚度矩阵时, 因为 $a_{i j}$ 是光滑的, 区域形状也很简单, 用少许几个点 即可得很精确的结果.

\section{四、数 值 例 子}

我们在一个 $L$ 形区域上(图 3) 求下列边值问题的数值解:

$$
\begin{aligned}
& -\nabla(a(x) \nabla u)=f, \\
& \left.u\right|_{\partial \Omega}=g .
\end{aligned}
$$

以 $(r, \theta)$ 记极坐标, 取 $a(x)=1+\frac{r^{2}}{8}$, 则 $-\nabla(a(0) \nabla)=-\Delta$. 再取 $u=r^{\frac{2}{3}} \sin \frac{2}{3} \theta+$

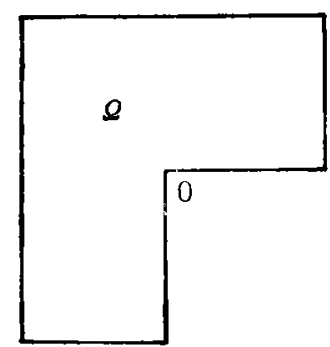

图 3

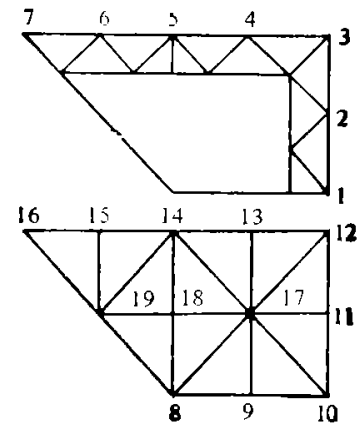

图 4 
$\frac{1}{8} x_{1}^{2} x_{2}^{2}$, 其中第一项是 Laplace 方程的一个奇性解, 它在零点的邻边上等于零, 当 $r \rightarrow 0$, 它的 微商与 $r^{-\frac{1}{3}}$ 同阶. 第二项是我们有意识地添加上去的, 使 $\boldsymbol{u}$ 不是 Laplace 方程的解. 这时

$$
f=-\frac{1}{6} r^{\frac{2}{3}} \cos \frac{49}{3}-\frac{r^{2}}{4}\left(1+\frac{r^{2}}{8}\right)-\frac{x_{1}^{2} x_{2}^{2}}{8} .
$$

由对称性, 只需计算 $\Omega$ 的一半. 我们作无限元与有限元两套网格如图 4, 其中取 $\xi=0.7$. 我们在 SX-386 微机上进行了计算, 得到无限元解为(按节点顺序):

$$
\begin{aligned}
& 0,0.1796,0.3838,0.3822,0.4440, \\
& 0.5505,0.7052,0,0,0, \\
& 0.1793,0.3712,0.3739,0.4200,0.5333, \\
& 0.6797,0.1900,0.2562,0.4018
\end{aligned}
$$

把两套网格上的解迭加以后, 在 $17,18,19$ 三点与精确解比较, 误差为(精确解减近似解):

$$
0.0018,0.0046,-0.0173
$$

精确解的变化区间为 $\left[0,2^{\frac{1}{3}}+\frac{1}{8}\right] \doteq[0,1.3849]$. 将最大误差与最大模比, 相对误差仅 $1.2 \%$ 左右. 在误差最大的第 19 节点处, 精确解为 $2^{-\frac{1}{3}}+\frac{1}{128} \doteq 0.8015$, 局部的相对误差也仅 $2 \%$ 左右. 注意到我们计算时仅用了 19 个节点,这个精度是很高的.

在计算数值积分时, 用梯形公式逐次加密的办法, 控制误差为 $10^{-4}$, 控制迭代次数为 7 次. 这样, 仍然有一些积分的误差没有达到 $10^{-4}$, 最大的误差为 $1.89 \times 10^{-3}$. 如果数值积分的计 算进一步精牉化, 解的精度还可望进一步提高.

为了作出对比, 用图 4 的有泿元网格同时作了计算。由于解有奇性, 网格又十分粗, 在 17 , 18,19 三点的误差很大, 甚至达到与解同样的数量级, 分别为

$$
-0.1188,-0 . j 116,-0.00251 \text {. }
$$

最大的相对误差达 $62 \%$ 。按相对误差比较, 无限元方法在这三点上的精度约为有限元方法的 31 倍.

\section{参考文献}

[1] Thatcher, R. W., Numer. Math., 25(1976), i63--178.

[2] 应隆女, 中国犁学, 1977,6：517-535.

[. 3$]$ Ying, L. A., J. Comp. Math., 4(1986), 2; $111-120$.

14] - Proceed 1 ngs of the Chind-France Symposium on FEM, Science Press, Beijing, China, Gordon and Breach, Science Publishers Inc., New York, 198;,542-565.

[5] — J. Comp. Math, 1(1983), 2: i30 -142.

[6] Kellogg, R. B., Singularities in Interface Problems, Numerical Solution of Partial Ditforential Euuations-ll, Academic Press. New York, London, 1971, 351--460.

[7] 韩厚德、这隆安, 计算数学, 1(1979), 1: 91-99.

[8] 许进超、应隆安, 同上,8(1986), 2: 164-174。

[9] Ciarlet, P. G., The Finite Element Method for Elliptic Problems, North-Holland, 1978.

[10] 应隆安, 应用数学学报, 2(1979),2: 149-166. 